\title{
A conceptual investigation of process controls upon flood frequency: role of thresholds
}

\author{
I. Struthers ${ }^{1}$ and M. Sivapalan ${ }^{2,3}$ \\ ${ }^{1}$ School of Environmental Systems Engineering, The University of Western Australia, 35 Stirling Highway, Crawley WA \\ 6009, Australia \\ ${ }^{2}$ Centre for Water Research, The University of Western Australia, 35 Stirling Highway, Crawley WA 6009, Australia \\ ${ }^{3}$ now at: Department of Geography and Department of Civil \& Environmental Engineering University of Illinois at \\ Urbana-Champaign, Urbana, Illinois, IL 61801, USA
}

Received: 11 October 2006 - Published in Hydrol. Earth Syst. Sci. Discuss.: 26 October 2006

Revised: 15 May 2007 - Accepted: 8 June 2007 - Published: 6 July 2007

\begin{abstract}
Traditional statistical approaches to flood frequency inherently assume homogeneity and stationarity in the flood generation process. This study illustrates the impact of heterogeneity associated with threshold non-linearities in the storage-discharge relationship associated with the rainfall-runoff process upon flood frequency behaviour. For a simplified, non-threshold (i.e. homogeneous) scenario, flood frequency can be characterised in terms of rainfall frequency, the characteristic response time of the catchment, and storm intermittency, modified by the relative strength of evaporation. The flood frequency curve is then a consistent transformation of the rainfall frequency curve, and could be readily described by traditional statistical methods. The introduction of storage thresholds, namely a field capacity storage and a catchment storage capacity, however, results in different flood frequency "regions" associated with distinctly different rainfall-runoff response behaviour and different process controls. The return period associated with the transition between these regions is directly related to the frequency of threshold exceedence. Where threshold exceedence is relatively rare, statistical extrapolation of flood frequency on the basis of short historical flood records risks ignoring this heterogeneity, and therefore significantly underestimating the magnitude of extreme flood peaks.
\end{abstract}

\section{Introduction}

Flood frequency analysis is concerned with describing the probabilistic behaviour of flood peaks for a given location; specifically, it attempts to quantify the likelihood of a flood

Correspondence to: M. Sivapalan

(sivapala@uiuc.edu) of a given magnitude occurring within a given time interval (Pattison et al., 1977). An understanding of flood frequency is of direct applicability for flood forecasting, but is most commonly used for engineering design against risk; for the design of flood-managing structures, such as reservoirs, drainage systems and levees, as well as flood-impacted structures, such as roads, bridges and buildings.

Traditional methods of flood frequency analysis rely on statistical fitting of historical flood records, where these are available. Where historical records are short, or the catchment is ungauged, flood frequency at high return periods is estimated either by extrapolation of statistical fits, or by regionalisation methods, in which the flood frequency behaviour of "similar" catchments are used to infer (usually involving some scaling relationship) the flood frequency behaviour of an ungauged or data-poor catchment (e.g. Gupta et al., 1996). Central to traditional statistical methods for flood frequency are the assumptions of homogeneity (i.e. that all flood peaks are independent, and drawn from the same probability distribution) and stationarity (i.e. that the probability distribution of flood peaks is temporally invariant). Land use change, including the construction of dams and levees, have long been acknowledged as violating the assumption of stationarity, such that flood frequency must be re-determined whenever the properties of the rainfall to flood transformation are altered due to changes in the catchment. More recently, non-stationarity due to the impact of climate variability and climate change have also been a subject of study (e.g. Kiem et al., 2003). The consequence of non-stationarity is that the flood frequency curve varies with time, such that the return period associated with a flood of a given magnitude does not have a fixed value, and loses its traditional meaning.

Published by Copernicus Publications on behalf of the European Geosciences Union. 
Less attention has been given to an examination of the impact of heterogeneity upon altering flood frequency. Heterogeneity is most commonly associated with a multiplicity of mechanisms responsible for a flood peak, whether these relate to heterogeneity in rainfall generation, or in the transformation of rainfall into a flood peak. Merz and Blöschl (2003) identified different storm types as resulting in different flood responses, with different storm types being important in describing different ranges of return period within the flood frequency curve. The consequence of heterogeneity is that, while the flood frequency curve does not vary with time, the flood frequency curve consists of different "regions" associated with different mechanisms or combinations of mechanisms that cannot be readily fitted by a simple probability distribution function. Where heterogeneity or non-stationarity is significant, therefore, traditional statistical methods for flood frequency analysis are unsuited.

An alternative, derived flood frequency approach attempts to apply our understanding of rainfall generation behaviour, and of the transformation process from rainfall into flood response, in order to understand the dynamics of flood frequency and its process controls (e.g. Eagleson, 1972). Unlike statistical methods, an understanding of process controls upon flood frequency permits the forecasting of flood frequency subject to non-stationarity and heterogeneity, as well as a rationalisation of non-stationarity and heterogeneity in historic flood frequency behaviour - at least qualitatively, if not quantitatively. The objective of this study is to present an analysis of the process controls on flood frequency for a simplified representation of catchment hydrological response, and then to illustrate the impact of heterogeneity in the hydrological response - specifically, that associated with threshold non-linearities in the catchment storage-discharge relationship - in altering flood frequency behaviour. The impacts of specific elements of spatial and temporal variability upon flood frequency are then considered. While Kusumastuti et al. (2006) considers the sensitivity of flood frequency to threshold impacts for a specific climate using similar climate and catchment parameterisations, this study aims to describe climate and catchment process controls upon flood frequency more generally, and to identify important parameter groupings that can be used to generally characterise the flood frequency response, including heterogeneity therein, of a given climate-catchment combination.

\section{Methodology}

This study couples a stochastic rainfall generator with a spatially-lumped rainfall-runoff model, which are run continuously for 1000 years in order to obtain estimates of flood frequency based on the maximum hourly flood intensity in each individual year of simulation (i.e. annual maxima series). Attenuation due to runoff routing in the river network is not explicitly considered. Our analysis employs "essen- tial" models, which contain only the basic elements of climate forcing and catchment hydrological response; evaporation is essentially continuous, except during rainfall, while the essential properties of rainfall are its intermittency and its stochasticity. The fundamental "action" of the catchment is to facilitate attenuation and loss, giving rise to a flood response signal which is filtered relative to the rainfall forcing signal. The various simplifications employed in this study make this methodology unsuitable for accurate flood prediction, but have the over-riding benefit - given the study objectives - of permitting the derivation of a relatively clear explanatory framework for the resulting flood frequency behaviour in terms of dominant climate and landscape properties.

\subsection{Stochastic rainfall generator}

Flood frequency is stochastic by its nature; in the absence of substantial historical precipitation records, the use of a stochastic rainfall generator is therefore logical for flood frequency examination. This study employs an event-based rainfall model similar to that of Robinson and Sivapalan (1997). In an event-based model, each "event" consists of a storm period of duration $t_{r}$ and average rainfall intensity $i$ and an inter-storm period of duration $t_{b}$. While Robinson and Sivapalan (1997) considered storm intensity to be correlated to storm duration, in this study each of these properties is considered to be an independent, exponentially-distributed random variable, such that:

$$
f_{X}(x \mid \hat{x})=\frac{1}{\hat{x}} \exp \left(-\frac{x}{\hat{x}}\right)
$$

where $x$ is the storm property variable $\left(t_{r}, i\right.$, or $\left.t_{b}\right)$, and $\hat{x}$ is an average value for that variable, and noting that $E[x]$ terms in certain figures and expressions are equivalent to $\hat{x}$. Withinstorm patterns in rainfall intensity for a given storm are generated using a stochastic disaggregation method, with identical parameterisation and methodology as given in Robinson and Sivapalan (1997). Seasonality in storm properties is initially ignored.

\subsection{Conceptual catchment model}

The catchment is represented as a single bucket (e.g. Manabe, 1969) of total storage capacity, $S_{b}$, and field capacity storage, $S_{f c}$. At storages above field capacity the subsurface flow rate is linearly related to catchment storage, modulated by a characteristic response time, $t_{c}$, such that:

$q_{s s}(t)=\left\{\begin{array}{cc}\frac{S(t)-S_{f c}}{t_{c}} & S(t)>S_{f c} \\ 0 & S(t) \leq S_{f c}\end{array}\right.$

Total storage capacity and field capacity storage can be directly related to catchment average soil depth, average porosity and moisture content at field capacity, while the characteristic response time can be estimated by calibration using as little as 5-10 days of streamflow data (Atkinson et al., 2002). 
Table 1. Parameter values used in the construction of figures. V indicates parameters which can be freely varied. AG indicates parameters that vary "as given" in the legend between individual curves, and N/A indicates variables which are not applicable to the particular figure.

\begin{tabular}{lccccccccl}
\hline Figure & $\hat{t}_{r}$ & $\hat{t}_{b}$ & $\hat{i}$ & $t_{c}$ & $S_{b}$ & $r_{A}$ & $r_{B}$ & $\beta$ & Other \\
\hline 1a, b & $\mathrm{V}$ & $\mathrm{V}$ & $\mathrm{V}$ & $\mathrm{N} / \mathrm{A}$ & $\mathrm{N} / \mathrm{A}$ & $\mathrm{N} / \mathrm{A}$ & $\mathrm{N} / \mathrm{A}$ & $\mathrm{N} / \mathrm{A}$ & \\
2a & 10 & 100 & 1 & $\mathrm{AG}$ & $\infty$ & 0 & $\mathrm{~N} / \mathrm{A}$ & 0 & \\
2b & $\mathrm{V}$ & $\mathrm{V}$ & $\mathrm{V}$ & $\mathrm{V}$ & $\infty$ & 0 & $\mathrm{~N} / \mathrm{A}$ & 0 & \\
$3 \mathrm{a}, \mathrm{b}, \mathrm{c}, \mathrm{d}$ & $\mathrm{V}$ & $\mathrm{V}$ & $\mathrm{V}$ & $\mathrm{V}$ & $\infty$ & $\mathrm{AG}$ & $\mathrm{AG}$ & 0 & \\
$3 \mathrm{e}$ & 10 & 100 & 1 & 100 & $\infty$ & $\mathrm{V}$ & $\mathrm{V}$ & 0 & \\
$4 \mathrm{a}$ & 10 & 100 & 1 & 100 & $\mathrm{~V}$ & 2 & 5 & 0 & \\
$4 \mathrm{~b}$ & $\mathrm{~V}$ & $\mathrm{~V}$ & $\mathrm{~V}$ & $\mathrm{AG}$ & $\mathrm{V}$ & $\mathrm{AG}$ & $\mathrm{AG}$ & 0 & \\
5 & 10 & 100 & 1 & 100 & 200 & 2 & 5 & 0 & \\
& & & & & & & & & $\begin{array}{l}t_{r a}=6 \mathrm{~h} \\
t_{b a}=50 \mathrm{~h}\end{array}$ \\
6 & 10 & 100 & 1 & 100 & 300 & 2 & 5 & 0 & $i_{a}=0.4 \mathrm{~mm} / \mathrm{hr}$ \\
& & & & & & & & & $e_{p a}=0.8 \mathrm{~mm} / \mathrm{hr}$ \\
$7 \mathrm{a}$ & 10 & 100 & 1 & 100 & $\mathrm{~V}$ & $\mathrm{AG}$ & $\mathrm{AG}$ & $\mathrm{AG}$ & \\
$7 \mathrm{~b}$ & 10 & 100 & 1 & 100 & 200 & 2 & 5 & $\mathrm{AG}$ & \\
\hline
\end{tabular}

During a storm event, evaporation is considered to be negligible, with rainfall infiltration constrained only by the storage capacity of the catchment. When the catchment is full, surface runoff occurs at a rate equal to the rainfall rate minus the ongoing subsurface flow rate;

$q_{s}(t)=\left\{\begin{array}{c}\max \left[i(t)-q_{s s}(t), 0\right] S(t)=S_{b} \\ 0 \quad S<S_{b}\end{array}\right.$

Thus, the equation for the rate of change of storage during a storm event is:

$\frac{d S(t)}{d t}=i(t)-q_{s s}(t)-q_{s}(t)$

So long as storages are above field capacity, subsurface flow is continuous during the inter-storm period, while surface runoff will cease. Evaporation is considered to occur during the inter-storm period at potential rates for storages greater than field capacity, and at a linearly-reduced rate below field capacity;

$e(t)= \begin{cases}e_{p}(t) & S(t) \geq S_{f c} \\ e_{p}(t) \frac{S(t)}{S_{f c}} & S(t)<S_{f c}\end{cases}$

such that the rate of change of storage during the inter-storm period can be expressed as:

$\frac{d S(t)}{d t}=-e(t)-q_{s s}(t)$

Unlike storm properties, the potential evaporation rate is not considered to be stochastically variable; seasonality in $e_{p}$ is also initially ignored, such that the value is constant during all interstorm periods.

\section{Results and discussion}

In attempting to summarise modelled flood frequency behaviour, it is useful to consider certain "end-member" limiting cases, the behaviour of which is in some ways simplified relative to the full complexity of the response. A trivial end-member is the case where storage is always below field capacity, such that no flood response occurs; obviously this case is of no consequence for flood frequency modelling. Two more meaningful end-member cases will be considered in turn: (i) Case 1 (maximum): an impervious catchment (i.e. $S_{b}=0$ ), for which all rainfall is transformed into surface runoff, and (ii) Case 2 (baseline): attenuation without thresholds; specifically, a deep soil with no evaporation, such that storage is always greater than field capacity but less than the total storage capacity, for which subsurface runoff will be continuously produced. Each of these end-member cases are themselves homogeneous; once these homogeneous cases are understood, we can then generalise to consider heterogeneity associated with evaporation below field capacity and intermittent saturation. In what follows, the expected value of the annual flood peak is denoted by $Q_{a v}$; numerical subscript refers to a flood peak associated with a given return period, e.g. the 1 in 50 year flood peak is denoted as $Q_{50}$; and superscripts denote flood peaks associated with a given end-member case. Parameterisations used in the construction of specific figures are summarised in Table 1.

Figures presented in this paper utilise estimated values of the average annual flood peak, and flood peaks for specified return periods. Each datapoint in Figs. 1a, b, 2a, 3a, $\mathrm{b}, \mathrm{c}, \mathrm{d}, 4 \mathrm{a}$, and $7 \mathrm{a}$ is obtained by analysis of the output of a 1000 year simulation, with different datapoints relating to different catchment or average climate parameter values, as described in Table 1. The remaining figures present flood 


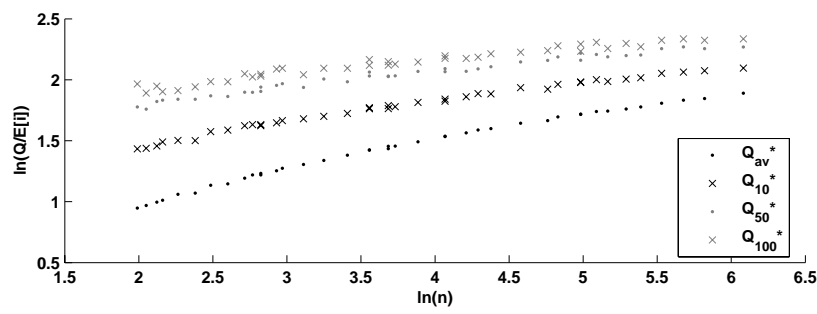

Fig. 1a. Rainfall frequency $\left(Q_{T}^{\max }\right)$ indices for the case without within-storm variability in storm intensity. Shown are the expected value of the rainfall intensity peak, the 1 in 10 year peak, 1 in 50 year peak and 1 in 100 year peak, for an assortment of rainfall parameterisations.

frequency curves, with each individual curve corresponding to a single simulation. As is always the case with flood frequency, uncertainty increases with increasing return period, such that replication with a different random number seed is capable of producing different flood frequency curves - especially at high return periods; while predictions may therefore be quantitatively different for each 1000 year realisation, the qualitative features of the output will be the same. For this reason, results from only one realization are presented in this paper. The primary motivation of this study is to understand process controls upon flood frequency, such that a majority of the effort in data analysis involved the mostly empirical identification of parameter groupings which could be used to "collapse" the full variety of flood response behaviour into singular curves with understandable behaviour; parameter groupings used as the $\mathrm{x}$-ordinate in many of the figures in this study represent the end-result of such analysis.

\subsection{Examination of "rainfall frequency" behaviour $\left(Q^{\max }\right)$}

Runoff and rainfall are intimately linked; the action of a catchment is essentially to filter and attenuate the rainfall signal to produce a runoff signal. In attempting to understand process controls upon flood frequency, an understanding of the stochastic nature of rainfall is therefore crucial. In the extreme case of a catchment that insignificantly filters and attenuates rainfall (e.g. a small car park or an initially very wet catchment), the runoff peak will be approximately the same as the rainfall intensity peak. In terms of the model employed in this study, this refers to the case where the total storage capacity, $S_{b}=0$, as well as the no evaporation cases where $t_{c}$ is infinitely large (i.e. all rainfall is converted to surface runoff) or else extremely small (i.e. all rainfall is converted immediately to subsurface runoff). Flood frequency analysis, specifically the annual exceedence probability, is concerned with peak intensities occurring in each year. Since all storm properties are considered independent, the magnitude of the largest storm intensity in a given year will be dependent upon the properties of the probability distribution function of storm intensity as well as the number of storms

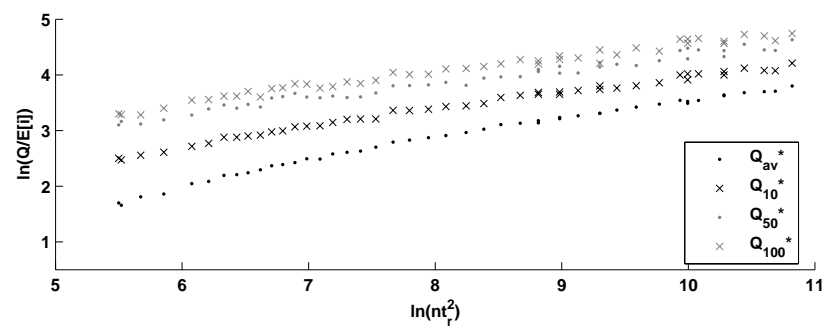

Fig. 1b. Rainfall frequency $\left(Q_{T}^{\max }\right)$ indices including within-storm variability in storm intensity. Shown are the expected value of the rainfall intensity peak, the 1 in 10 year peak, 1 in 50 year peak and 1 in 100 year peak, for an assortment of rainfall parameterisations.

(i.e. number of random realisations) in the year; the average number of storms in a year is given by:

$n=\frac{365 \times 24}{\hat{t}_{r}+\hat{t}_{b}}$

The exponential distribution for storm intensity has only one parameter, the average storm intensity $\hat{i}$, which acts as a scaling factor upon the distribution of storm intensity values; by scaling the rainfall intensity peaks by $\hat{i}$, Fig. 1a shows that (for the case ignoring within-storm variability in storm intensity) the expected value of the scaled annual rainfall intensity peak, as well as scaled rainfall intensity peaks associated with any given return period, will increase as the average number of storm events in a year increases, as expected. Specifically, the average value of the annual rainfall intensity peak obtained through simulations with the stochastic rainfall model (without within-storm variability) was found to fit the following empirical expression:

$Q_{a v}^{\max }=1.42 \hat{i} n^{0.845}$

This figure is essentially a representation of the extreme value distribution of average storm intensity; for the assumed exponential parent distribution function for storm intensity, the extreme value distribution will exactly fit a Gumbel distribution (Sivapalan and Blöschl, 1998). The addition of within-storm variability in storm intensity adds an additional dependency upon the average storm duration itself (Fig. 1b); this is due to the fact that the number of bisections necessary to resolve within-storm variability to a specified resolution (e.g., hourly) is directly functional upon storm duration. It is crucial to note, however, that the relative impact of the average storm duration upon resulting peak intensity behaviour, represented by the exponent value of 2 , is not necessarily a general finding; alternative parameterisations and temporal resolutions for rainfall disaggregation may result in different exponents, if not completely different functional forms, possibly incorporating parameters relating to the disaggregation of rainfall to fine scales. Nonetheless, for the specific parameterisation employed in this study, the average annual rainfall 


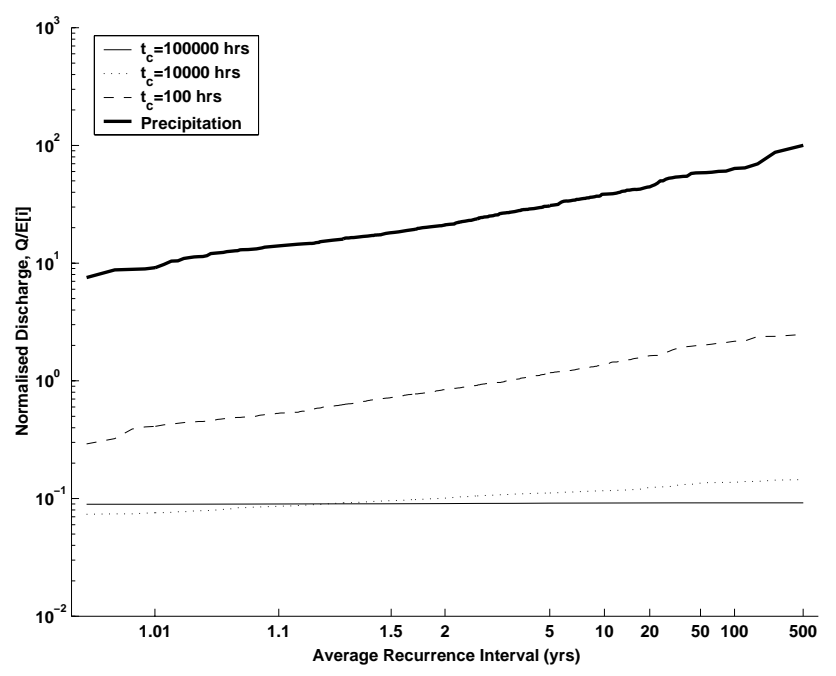

Fig. 2a. The impact of the characteristic response time for subsurface flow upon baseline flood frequency.

intensity peak including the impact of within-storm variability was found to fit the following empirical expression:

$Q_{a v}^{\max }=0.031 \hat{i}\left(n \hat{t}_{r}^{2}\right)^{3.04}$

Similar relationships, with different numerical coefficients but of the same functional form, can be derived relating to peaks for specified return periods, such as those presented in Figs. 1a and b. Of course, these empirical relationships are valid for the specific assumptions made in relation to rainfall stochasticity; deviation of storm duration, inter-storm period or average storm intensity from the assumed exponential distributions, correlation between storm duration and average intensity, and systematic long-term variability in storm properties (associated with specific climate variability cycles, for example), are among the possible complexities which may lead to actual rainfall frequency deviating from these predictions. Seasonal variability in average storm properties will, of course, also have an impact; this will be considered later. Note that all simulations conducted hereinafter include within-storm variability in storm intensity.

3.2 Flood frequency ignoring thresholds and evaporation $\left(Q^{\text {base }}\right)$

We initially consider the simplified no evaporation case with an infinitely large storage capacity; for this scenario subsurface flow is continuous and is the sole runoff generation mechanism. We will herein refer to this scenario as the "baseline" case, i.e. the flood frequency response as impacted by the basic intermittency of storms, and attenuation by the catchment. Figure 2a illustrates the impact that the characteristic response time for subsurface flow, $t_{c}$, has upon flood frequency for a particular climate for the baseline case. For

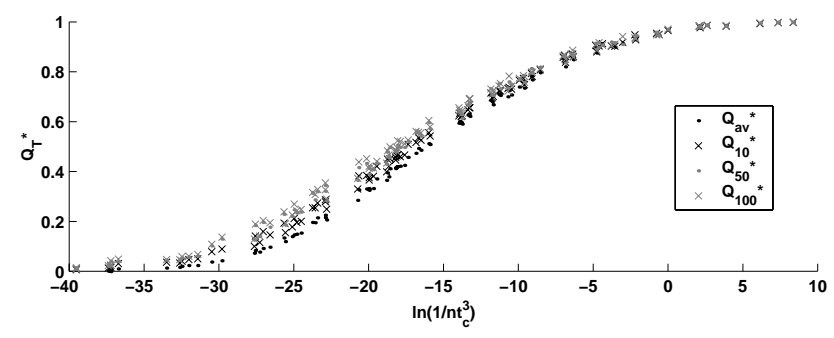

Fig. 2b. Baseline flood frequency indices for an assortment of storm and catchment parameterisations.

the sake of comparison we also present $Q_{T}^{\max }$ in this figure (representing essentially the rainfall peak). For extremely slow subsurface flow, as $t_{c} \rightarrow \infty$, stochastic variability in the annual flood peak is negligible, making the flood frequency curve essentially flat. From analysis conducted using a range of climate properties, this minimum value of flood peak for the baseline case is found to be independent of $T$ and to be approximately equal to:

$Q^{\min }=\hat{i}\left(\frac{\hat{t}_{r}}{\hat{t}_{r}+\hat{t}_{b}}\right)$

Faster subsurface flow, associated with lower values of $t_{c}$, causes a steepening of the flood frequency curve, as runoff behaviour becomes more responsive to stochasticity in rainfall behaviour. As we have previously identified, as $t_{c} \rightarrow 0$, flood frequency will converge with rainfall frequency, $Q_{T}^{\max }$ (corresponding to the rainfall peak). We investigated whether the "baseline" behaviour for specified $t_{c}$ values between falling these extremes can therefore be expressed compactly in terms of $Q^{\min }, Q_{T}^{\max }$ and the specific value of $t_{c}$. Figure $2 \mathrm{~b}$ illustrates the end result of this exploration for a wide range of average storm properties and values of $t_{c}$. Note that the y-ordinate in this and many subsequent figures is a normalised value of the natural logarithm of the flood peak, given by:

$Q_{T}^{*}=\frac{\ln \left(Q_{T}^{\text {base }}\right)-\ln \left(Q^{\min }\right)}{\ln \left(Q_{T}^{\max }\right)-\ln \left(Q^{\min }\right)}$

where $T$ refers to the return period of interest. The expression for the $\mathrm{x}$-ordinate in Fig. $2 \mathrm{~b}$ (and many subsequent plots) is dominated by the impact of $t_{c}$, such that we can generalise it to describe increasing values of $\mathrm{x}$ to correspond to faster subsurface flow response time (i.e. lower $t_{c}$ ). From Fig. $2 \mathrm{~b}$ it is apparent that the scaled flood peak for the baseline case takes the form of a simple logistic function with respect to the natural logarithm of the parameter group $n t_{c}^{3}$. The resulting empirical functional form describing the curves presented in Fig. $2 b$ is therefore of the form:

$$
Q_{T}^{*}=\frac{1}{1+a\left(n t_{c}^{3}\right)^{b}}
$$




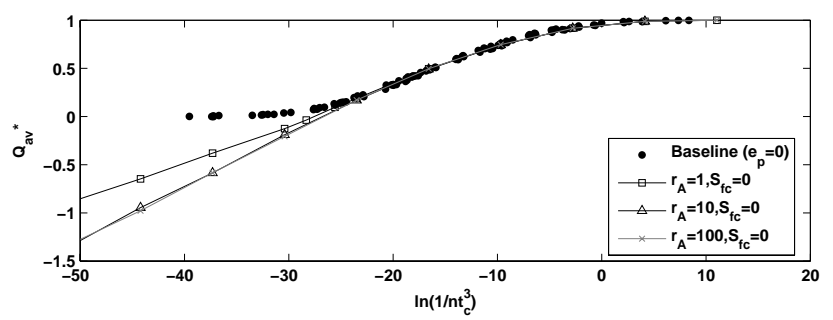

Fig. 3a. Expected value of the annual flood peak, for the nonthreshold case including evaporation for different values of the dimensionless ratio, $r_{A}$.

where $a$ and $b$ are empirical coefficients that vary only slightly depending upon $T$ (i.e. $a=0.0519$ and $b=-0.174$ for $T=10$ years, compared to $a=0.0534$ and $b=-0.163$ for $T=100$ years). Allowing for the scatter associated with the finite simulation length - especially in relation to large return periods - these curves approximately overlap, such that variation in $a$ and $b$ with $T$ is relatively small, i.e. $Q_{T}^{*}$ is approximately constant for a given value of $n t_{c}^{3}$. We can regard $Q_{T}^{*}$, which ranges in value from 0 to 1 , as an index of the slope of the flood frequency curve relative to the rainfall frequency curve. A value of $Q_{T}^{*}$ approaching 0 corresponds to $t_{c} \rightarrow \infty$, with flood peaks due to subsurface flow being relatively invariant from one year to another, approximately equal to $Q^{\mathrm{min}}$. As $Q_{T}^{*}$ increases, flood peaks due to subsurface flow will be increasingly impacted by eventscale variability in storm properties (and consequent eventscale variability in the antecedent condition), such that flood magnitude increases significantly with increasing return period. As $Q_{T}^{*}$ approaches 1, attenuation by the catchment becomes minimal, and the flood frequency curve has the same slope as (and is therefore equivalent to) the rainfall frequency curve. In summary, the slope and magnitude of the baseline flood frequency curve is functional upon the average values of storm intensity, storm duration, and number of storms per year, which together determine the magnitude of $Q_{T}^{\max }$, and the characteristic response time for subsurface flow, which determines the degree of attenuation associated with the rainfall to runoff transformation.

3.3 Impact of evaporation on subsurface flow flood frequency

In terms of the rainfall to runoff transformation, evaporation acts essentially as a loss mechanism. If we consider evaporation during rainfall to be negligible, evaporation during inter-storm periods is capable of reducing the flood peak indirectly via its impact upon reducing antecedent storage prior to a given storm event. In the absence of a field capacity storage threshold, fast-draining soils (i.e. low $t_{c}$ ) will tend to dry towards zero storage by the end of inter-storm periods even without evaporation, such that the impact of evaporation

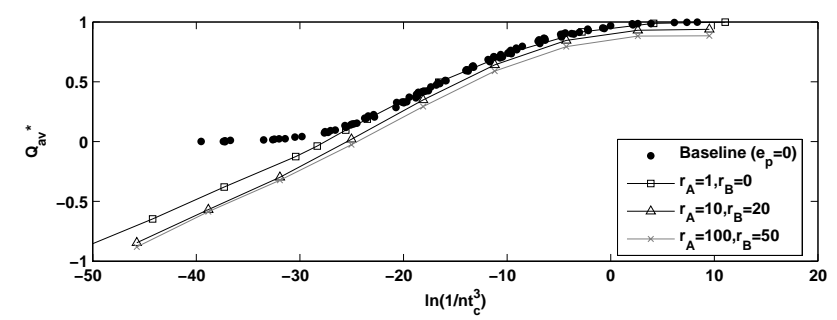

Fig. 3b. Expected value of the annual flood peak, for the case with field capacity threshold and evaporation for different combinations of the dimensionless ratios $r_{A}$ and $r_{B}$.

upon the antecedent condition and hence flood frequency will be negligible. In contrast, for slow-draining soils, the impact of evaporation in reducing the antecedent moisture content will be significant, such that the evaporation will have a noticeable impact upon flood frequency. Figure $3 \mathrm{a}$ presents the expected value of the annual flood peak in the absence of a field capacity threshold for various strengths of evaporation in contrast to the baseline (no evaporation) case. For values of the x-ordinate greater than -20 , which typically corresponds to values of $t_{c}<200 \mathrm{~h}$, evaporation has no impact, such that all curves overlap with the baseline curve. Only for $t_{c}>200 \mathrm{~h}$ is the soil sufficiently slow-responding that evaporative reduction in the antecedent condition causes a reduction in the average annual flood peak. The magnitude of the evaporative impact obtained from the simulations was found to be a function of the ratio of the per-event expected potential evaporation volume to the expected rainfall volume, $r_{A}=\frac{\hat{e}_{p} \hat{t}_{b}}{\hat{i}_{t_{r}}}$. Where this ratio is significantly greater than 1 , the behaviour of $Q_{a v}^{*}$ for x values less than -20 is approximately linear (on semi-log axes) and relatively unchanging. If the ratio is equal to 0 , the baseline expression describes its behaviour. Where $0<r_{A}<1$, the behaviour falls between these two "envelope" curves.

3.4 Impact of field capacity on subsurface flow flood frequency

For the no evaporation case, and for an infinite catchment storage capacity, the addition of a field capacity has no impact whatsoever upon flood frequency; it will merely modify the storage corresponding to a given subsurface flow rate, as given by (2). This was already reflected in the results presented in Figs. 2a and b. Only when combined with evaporation is the field capacity threshold capable of impacting runoff generation, and hence flood frequency. Specifically, the impact of evaporation in combination with field capacity is to permit the (intermittent) cessation of subsurface flow. Particularly in dry years, this will result in significant reductions in the magnitude of the annual flood peak; where flow cessation lasts for the entire year, the annual flood peak is 0 . The frequency and persistence of flow cessation was 


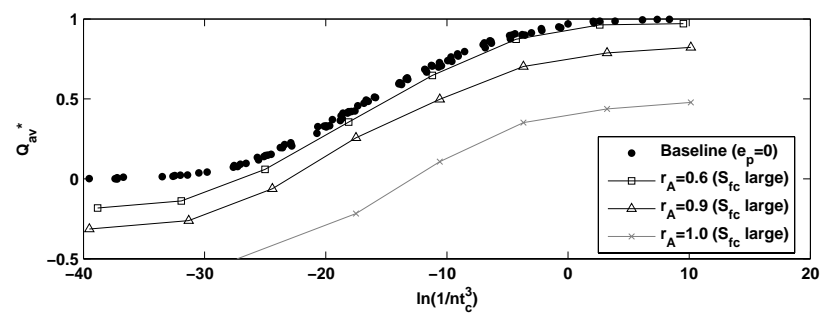

Fig. 3c. Expected value of the annual flood peak for the case of extremely large field capacity threshold and evaporation.

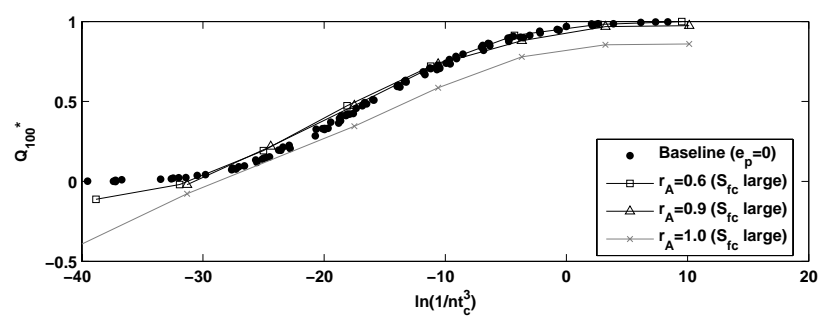

Fig. 3d. Predictions of the 100 year return period flood peak for the case of extremely large field capacity threshold and evaporation for different values of the dimensionless ratio $r_{A}$.

again found to depend upon $r_{A}$, as well as $r_{B}=\frac{S_{f c}}{\hat{e}_{p} \hat{t}_{b}}$, the ratio of field capacity storage to the event potential evaporation volume, which is a measure of the relative magnitude of potential storage deficit below field capacity. The role of evaporation is to reduce the antecedent storage to below the field capacity threshold and its impact upon flood frequency is maximised where these two ratios are large. Figure $3 \mathrm{~b}$ illustrates how the expected value of the annual flood peak for subsurface flow is modified from the baseline behaviour by the introduction of a field capacity storage threshold, in combination with evaporation. For $r_{A} \leq 1$, behaviour is essentially the same as the case without the field capacity threshold (i.e. Fig. 3a) unless $r_{B}>>1$. As $r_{B}$ increases, the impact of evaporation below field capacity is manifested as a downward translation of the curves relative to the "evaporation without field capacity" case for the same value of $r_{A}$.

Figure $3 \mathrm{c}$ shows the impact of evaporation for the case of an extremely large field capacity, $r_{B}>1 \times 10^{8}$; for this scenario, the ratio $S / S_{f c}$ in (5), remains approximately equal to 1 throughout the simulation, such that evaporation occurs at potential rates above and below field capacity. Contrasting this to Fig. $3 b$ illustrates the potential importance of the piecewise evaporation assumption in (5) upon resulting flood frequency behaviour. In this scenario, flood frequency behaviour is found to be highly sensitive to the value of $r_{A}$; for values significantly lower than 1 , the volume of evaporation is low relative to the storm volume, and the impact of evaporation is not significant. As $r_{A}$ increases towards 1 , the evaporative volume is comparable to the storm volume,

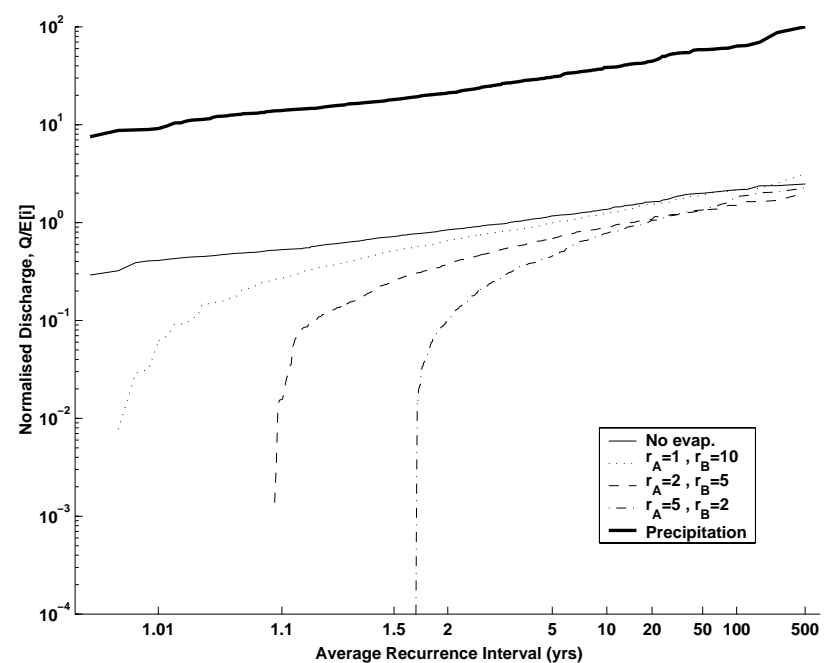

Fig. 3e. Impact of evaporation and field capacity threshold upon flood frequency for different combinations of the dimensionless ratios $r_{A}$ and $r_{B}$.

such that the runoff response is highly sensitive to stochastic variability in storm properties, and hence the antecedent storage condition. The impact of evaporation is less significant in wetter years associated with higher return periods; Fig. 3d shows the 100 year return period flood peak for the same cases as Fig. 3c. For applications specifically interested in extreme flood events only, therefore, accurate accounting of the complex impacts of evaporation upon flood frequency may not be particularly crucial, especially where $r_{A}<1$.

Figure 3e presents a partial summary of the impact of evaporation upon flood frequency, showing the impact of increasing the value of $e_{p}$ alone (which is manifested as a proportional increase in the value of $r_{A}$ and reduction in $r_{B}$ ). This figure provides further insights into the summary results presented above in Figs. $3 \mathrm{c}$ and d; higher evaporation relative to storm volume (i.e. higher $r_{A}$ ) for a moderately large field capacity results in an increased prevalence of flow cessation, with many years generating no flood response. But at larger return periods, the discrepancy between each curve reduces rapidly, until the impact of evaporation is minor for floods with an average recurrence interval of 100 years or more. Of course, the return period at which these curves converge is dependent upon the values of $r_{A}$ and $r_{B}$; where these values are larger, convergence could occur at return periods greater than 100 years.

\subsection{Impact of saturation}

Analysis thus far has assumed an infinite catchment storage capacity, such that saturation, and hence saturation excess surface runoff, cannot occur. A finite storage capacity introduces the possibility of saturation excess, which is a faster runoff response mechanism (i.e. considered here to have an 


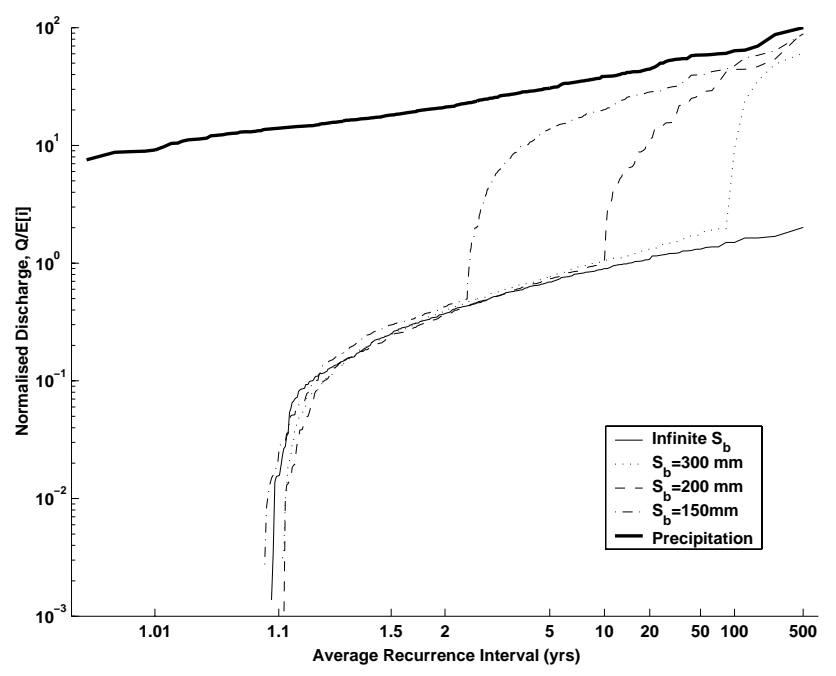

Fig. 4a. Impact of finite catchment storage capacity, and saturation excess, upon flood frequency.

instantaneous concentration time) than subsurface flow.

Figure 4a illustrates the impact of a finite catchment storage capacity and resulting saturation excess upon flood frequency in contrast to a catchment with infinite storage capacity, and hence no saturation excess. The onset of saturation excess results in a significant break in the flood frequency curve at a return period associated with the average recurrence interval between years in which saturation excess is triggered. Flood frequency at high return periods eventually converges towards the rainfall frequency curve (since surface runoff is assumed to be converted instantaneously to a flood response; a finite response time for surface runoff would result in peaks below the rainfall frequency values). The break in curve occurs at lower return periods as the catchment storage capacity reduces. At return periods below this break, flood peaks are associated with subsurface flow only, such that this region of the flood frequency curve is not impacted by changes in the value of $S_{b}$. Similar breaks or "transitions" in the flood frequency curve have previously been described by Sivapalan et al. (1990), associated with a transition from partial area saturation excess-dominated to infiltration excess-dominated runoff. Where several runoff mechanisms exist, the flood frequency curve may therefore have multiple breaks, each associated with a transition in the dominant runoff-producing mechanism.

Previous work by Struthers et al. (2007) formulated a dimensionless index of the relative frequency of saturation excess triggering for equivalent rainfall and runoff models as those used here:

$$
\alpha=\frac{\hat{i} t_{c}\left[\exp \left(\frac{\hat{t}_{r}}{t_{c}}\right)-1\right]}{\left(S_{b}-S_{f c}\right)\left[\exp \left(\frac{\hat{t}_{r}}{t_{c}}\right)-\left(\frac{\hat{S}_{0}-S_{f c}}{S_{b}-S_{f c}}\right)\right]}
$$

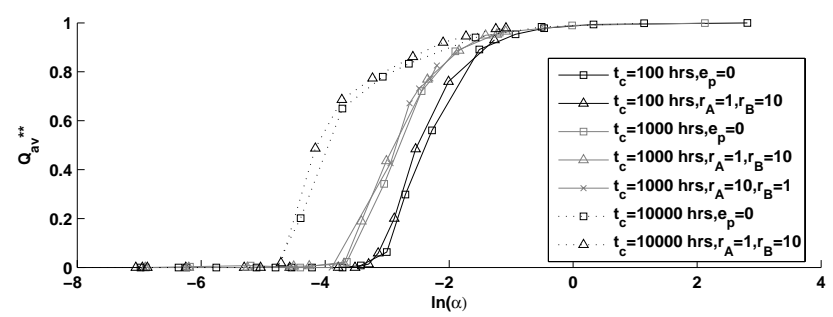

Fig. 4b. Example relationships between normalised values of the annual flood peak and the index $\alpha$ for different combinations of the dimensionless ratios $r_{A}$ and $r_{B}$.

where $\hat{S}_{0}$ is the expected value of antecedent storage, which in this study is determined a posteriori from analysis of simulation data. This index is a direct re-arrangement of the single-storm criteria for the triggering of saturation excess; for an individual storm with these average properties incident upon a catchment with average antecedent storage, saturation excess will occur only if $\alpha>1$. When applied instead to a population of stochastically varying storms, and hence stochastically varying antecedent conditions, $\alpha$ instead relates to the per-storm frequency of saturation excess occurrence, with this relative frequency increasing as $\alpha$ increases.

In the case of a sufficiently large catchment storage capacity, even an extreme duration and intensity storm occurring in wet antecedent conditions will be insufficient to generate saturation excess, and flood frequency behaviour is identical to the subsurface flow-only case considered thus far. In the opposite extreme, where the catchment storage capacity is sufficiently small that all storms trigger saturation excess, it is apparent from the summation of (2) and (3) that the annual flood peak will correspond to the annual rainfall intensity peak. Once more, it is therefore possible to normalise flood frequency behaviour between the subsurface flow only value, which we will denote as $Q^{s s}$, and the rainfall frequency value, $Q_{T}^{\max }$, for a given return period $T$ :

$$
Q_{T}^{* *}=\frac{\ln \left(Q_{T}\right)-\ln \left(Q_{T}^{s s}\right)}{\ln \left(Q_{T}^{\max }\right)-\ln \left(Q_{T}^{s s}\right)}
$$

Figure $4 \mathrm{~b}$ presents example cases of the impact of intermittent triggering of saturation excess, which is functional on $\alpha$, upon the expected value of the annual flood peak; for a given curve, variation in $\alpha$ is obtained by altering the bucket storage capacity, $S_{b}$ (noting that this will have a consequent impact in altering $\hat{S}_{0}$ ). As expected, below some threshold value of $\alpha$, wetting due to rainfall is insufficient to cause saturation, such that flood frequency is the same as the subsurface flow only case, $Q^{s s}$. As $\alpha$ increases, saturation excess may occur rarely, associated with long return periods only. Further increases in $\alpha$, associated with a wetter climate and/or a smaller catchment storage capacity, results in saturation excess occurring more often, but the likelihood of the timing of saturation excess corresponding with the timing of the peak 
annual rainfall intensity is still small, and therefore the flood frequency is still significantly below $Q_{T}^{\max }$. Only with further increases in $\alpha$ is the occurrence of saturation excess so common that, in most years, its occurrence is concurrent with the annual rainfall intensity peak, and flood frequency is approximately equal to the rainfall frequency. Note, however, that the return period at which the break in the flood frequency curve occurs does not necessarily correspond to the average time between saturation excess events; the importance of antecedent conditions upon saturation excess triggering means that its occurrence is often clustered in time, i.e. there is a relatively high probability of repeated triggering of saturation excess by storms immediately following its initial triggering. Due to clustering, in years when saturation excess occurs there is a finite probability that it will occur multiple times. As a consequence, the average time between saturation excess triggering (i.e. the average of the full distribution) is less than or equal to the return period associated with the break in the flood frequency curve (i.e. the average of the extreme value distribution). Nonetheless, increases in $\alpha$ above the lower threshold value are associated with a reduction in the average time between saturation excess triggering (as implied from the results of Struthers et al., 2007), as well as a reduction in the return period associated with the break in the flood frequency curve.

Unlike the description in relation to subsurface flow flood frequency, $Q^{s s}$, the behaviour of flood frequency including saturation excess is still not firmly defined; while behaviour is relatively consistent for a given value of $t_{c}$, the threshold value of $\alpha$ associated with the onset of saturation excess and the degree of curvature changes with changing magnitudes of $t_{c}$ in a manner that is as yet not understood. The index $\alpha$ is therefore imperfect, but is nonetheless useful in describing the basic process controls upon the frequency of saturation excess occurrence, and its consequent impact upon flood frequency.

\subsection{Summary}

For the simplified rainfall and runoff response assumptions used in this study, Fig. 5 presents a summary of the heterogeneity in the resulting flood frequency in terms of four regions, corresponding to (i) evaporation- and field capacitycontrolled flow reduction and cessation, (ii) storm intermittency and stochasticity-controlled subsurface flow, (iii) saturation excess surface flow limited by saturation triggering frequency, and (iv) rainfall-limited saturation excess surface flow. The transition between regions (i) and (ii) is usually gradual, associated with a reduction in the impact of evaporation upon flood peaks in wetter rainfall years. In contrast, the transition between regions (ii) and (iii) is abrupt, due to the significantly quicker response time associated with surface runoff compared to subsurface runoff, which results in order of magnitude changes in the resulting flood peak. Region (iii) refers to the situation where saturation excess is occurring,

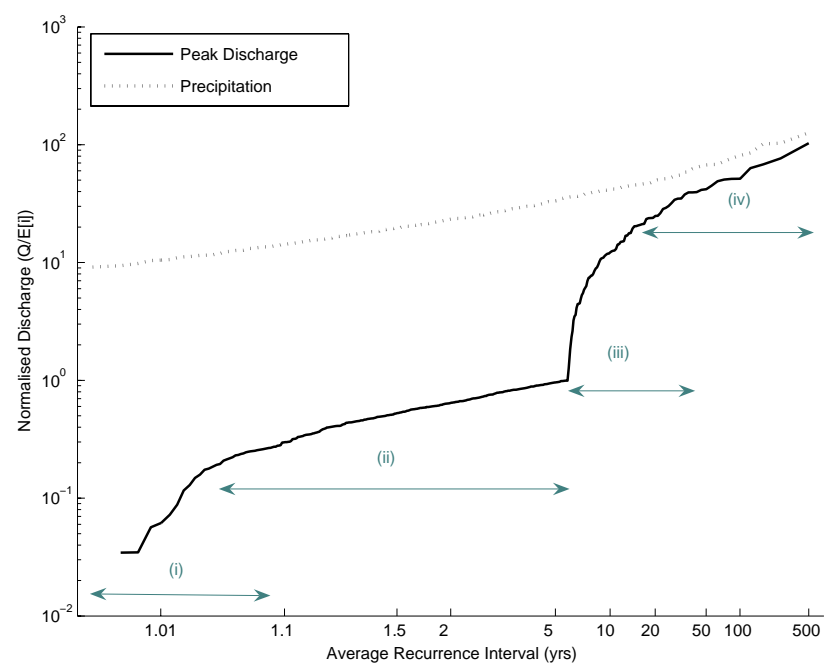

Fig. 5. Illustration of distinct regions of behaviour and process control within the modelled flood frequency curve.

but does not necessarily occur at the same time as extreme rainfall intensities in a given year, such that flood frequency peaks for a given return period are still significantly below the corresponding rainfall intensity peak. Rainfall frequency provides an upper limit for flood frequency, such that region (iv) contains values of annual flood peak caused by saturation excess which is concurrent with extreme rainfall intensities (i.e. intensities of the same order as the annual rainfall peak in a given year).

Each region essentially has distinct process controls and a very distinct curve shape, such that it would be difficult to fit behaviour with a simple statistical model without introducing significant inaccuracies. Given that statistical approaches are commonly used to obtain estimates of large return period flood magnitudes by extrapolation from short historical flood records, which may not incorporate rare events such as saturation excess overland flow, the benefit of a derived flood frequency approach over a statistical approach is readily apparent.

The framework developed in this study can be extended to consider the impact of additional complexities in altering the idealised flood frequency behaviour presented in Fig. 5. Seasonality has been ignored thus far, in order to reduce the number of parameters in the model, but will undoubtedly impact upon the magnitude of the annual flood peak. By conceptualising the catchment using a single bucket, the model assumes that overland flow is, in a sense, a binary process; occurring over the whole catchment, or not occurring at all. Of course, the more conventional and realistic approach to overland flow involves the consideration of contributing areas where, for a majority of time, some percentage of the catchment, greater than $0 \%$ but less than $100 \%$, is contributing to saturation excess overland flow. 


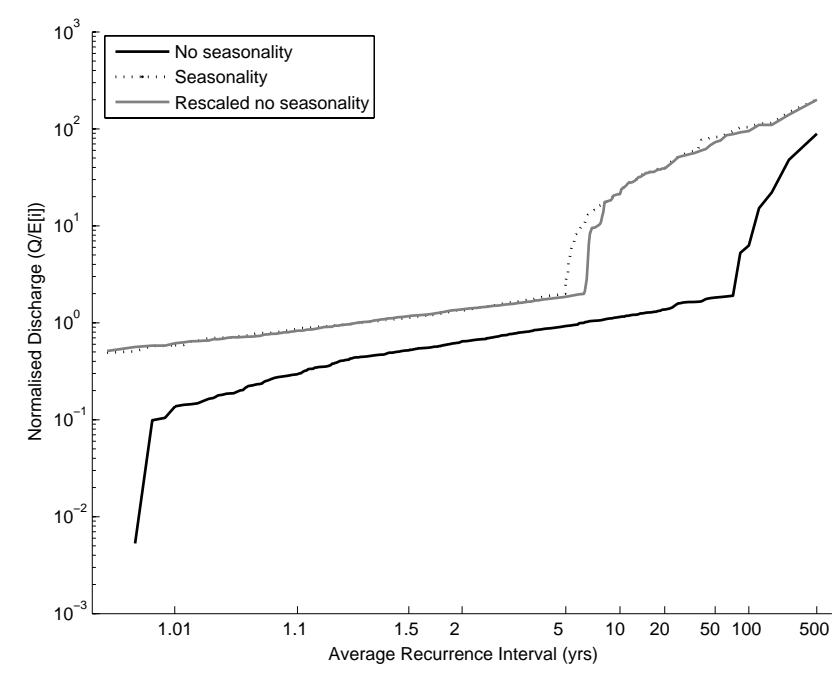

Fig. 6. The impact of seasonality upon flood frequency. Also illustrated is the ability to approximately account for simple seasonality implicitly using appropriately scaled average parameter values without seasonality.

\subsection{Impact of seasonality}

The analysis thus far considered the impact of threshold nonlinearities upon flood frequency in response to climate forcing with stochastic variability at within-storm and betweenstorm scales, but without deterministic variability associated with seasonality. It is beyond the scope of a conceptual study such as this to consider the full complexity of seasonal variability in storm and inter-storm properties associated with, for example, seasonal differences in storm type (e.g., synoptic versus cyclonic rainfall). We simply wish to illustrate the impact that seasonal variability in storm properties, evaporation, and hence antecedent conditions, could have upon flood frequency. Seasonality is incorporated in the rainfall generator using a simple sinusoidal variability in average storm and interstorm properties, of the form:

$\hat{x}_{t}=\bar{x}+x_{a} \sin \left\{\frac{2 \pi}{\omega_{h}}\left(t-t^{\prime}\right)\right\}$

where $\hat{x}_{t}$ is the average value of the property in question (i.e. storm duration, interstorm period, potential evaporation rate, average storm intensity), for use in (1), $\bar{x}$ is the sinusoidal mean value, $x_{a}$ is the seasonal amplitude, $t$ is the hour of the year, and $t^{\prime}$ is the phase lag in hours. As with the original (non-seasonal) methodology, the actual value of a given realisation of storm duration, interstorm period, and average storm intensity is considered to be stochastically variable (i.e. with $\hat{x}_{t}$ as the mean value of the stochastic distribution at time $t$ ), while potential evaporation is non-variable (i.e. with a value equal to $\hat{x}_{t}$ at time $t$ ). Our examination of seasonality impacts is restricted to the case of out-of-phase seasonality between "wetting" and "drying" climate elements; i.e. sea- sonality in storm duration and average storm intensity are considered to be perfectly in phase with one another, and of opposing phase to interstorm period and potential evaporation seasonality. This "wet season-dry season" simplification is reasonable for most non-tropical climates.

For flood frequency, which is concerned with annual extremes rather than the complete stochasticity of flood response, we would expect seasonality to have two types of impact: a direct impact, in terms of longer or more intense storms in a given season, leading to larger runoff response, and an indirect impact associated with seasonality in the antecedent condition resulting from seasonality in storm properties. Seasonality in antecedent moisture will be lagged to some degree relative to seasonality in storm properties, such that it probably will not simply be a case of applying the methodology previously described using "wet season" average climate parameters in place of seasonally-averaged climate parameters. Indeed, it was found that a given "wet season-dry season" scenario could be reasonably replicated by a "no seasonality" scenario in which the required parameter values (i.e. $\hat{t}_{r}, \hat{t}_{b}, \hat{i}$ and $e_{p}$ ) were obtained by modifying the sinusoidal average values as appropriate (i.e. increased for wetting climate elements, decreased for drying climate elements) by approximately $50 \%$ of the seasonal amplitude. In other words, a case in which $x_{a}$ is non-zero for a given property can be approximately replicated by a case in which $x_{a}$ is assigned a value of zero, and $\bar{x}$ is appropriately modified. For example, a climate with an $11 \mathrm{hr}$ sinusoidal mean storm duration with $4 \mathrm{hr}$ seasonal variability in this value can be approximately replicated by assuming a $13 \mathrm{~h}$ average storm duration without seasonal variability; seasonality in average storm intensity can be similarly accounted for. Conversely, if potential evaporation varies seasonally with a $0.1 \mathrm{~mm} / \mathrm{hr}$ sinusoidal average with $0.04 \mathrm{~mm} / \mathrm{hr}$ seasonal variability, this can be approximately replicated by using a value of $0.08 \mathrm{~mm} / \mathrm{hr}$ without seasonal variability; seasonality in the average interstorm period can likewise be accounted for.

Invariably, the impact of seasonality is to increase the flood peak associated with a given return period, relative to the same average climate without seasonality. More specifically, seasonality will decreases the frequency of, or eliminate altogether, flow cessation over an entire year. It will also increase the frequency of saturation excess triggering, such that the break of slope in the flood frequency curve will occur at lower return periods. In effect, the impact of seasonality is to translate the flood frequency curve to the left, such that floods of a given magnitude are associated with a lower return period relative to the no seasonality case. Figure 6 illustrates the impact of seasonality upon increasing the frequency of saturation excess (hence reducing the return period associated with the break in the flood frequency curve), reducing the frequency of or eliminating flow cessation, and increasing the magnitude of flood peaks associated with subsurface flow. Also illustrated is the ability of a non-seasonal simulation with appropriately modified parameter values to 


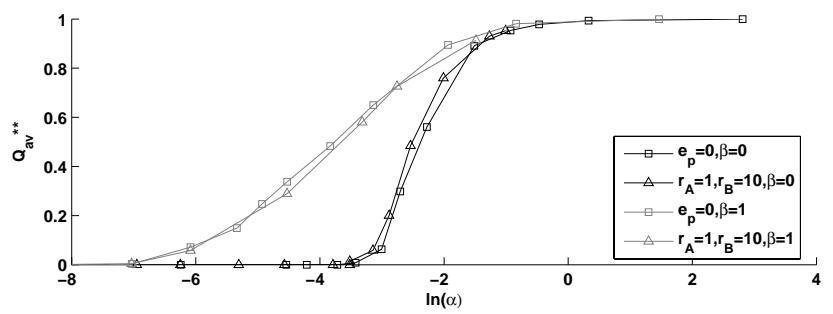

Fig. 7a. Impact of variable soil depth and partial saturation upon the $\alpha$ relationship with the normalised expected annual flood peak for different combinations of the dimensionless ratios $r_{A}$ and $r_{B}$ and the parameter $\beta$ of the Xinanjiang distribution.

reasonably replicate the flood frequency of a seasonallyvariable climate.

\subsection{Impact of landscape spatial variability}

A lumped representation of the catchment was employed in this study so far, which ignores the impact of spatial variability in the timing and intensity of rainfall, as well as spatial organisation in landscape properties. Issues of spatial scale and spatial variability are considered in detail in other studies (e.g. Blöschl and Sivapalan, 1997; Jothityangkoon and Sivapalan, 2001); this study restricts itself to considering the impact of simple spatial organisation in soil depth, and consequent spatial variability in threshold triggering, upon flood frequency. Soil depths are considered to vary with distance away from the stream according to the Xinanjiang distribution (Wood et al., 1992), where the depth of bucket $i$ in a series of $n$ buckets is given by:

$S_{b, i}=S_{\max }\left[1-\left(1-\frac{i-0.5}{n}\right)^{1 / \beta}\right]$

where $S_{\max }$ is effectively a scaling coefficient for all soil depths and $\beta$ is a shape parameter. Buckets are considered to be connected in series, from deepest to shallowest, with the characteristic response time for subsurface flow in each bucket, $t_{c, i}$, being equal. The impact of spatial variability in soil depth is assessed by comparing the obtained flood frequency against the flood frequency for a lumped catchment with the same average soil depth, i.e. $S_{b}=E\left[S_{b, i}\right]$, and with an equivalent catchment-scale characteristic response time for subsurface flow, $t_{c}=n t_{c, i}$. Note that the multiple bucket case with $\beta=0$ behaves identically to the single-bucket case with equivalent average soil depth and catchment-scale characteristic response time for subsurface flow, such that there is no discretisation artefact.

Figure 7a provides an example of the impact of spatial variability in soil depth upon the triggering of saturation excess; the value of $\alpha$ is calculated for the equivalent singlebucket case, but response behaviour relates to a variable soil depth. As for Fig. 4a, variation in $\alpha$ is obtained by altering the bucket storage capacity, $S_{b}$, and the onset of (partial)

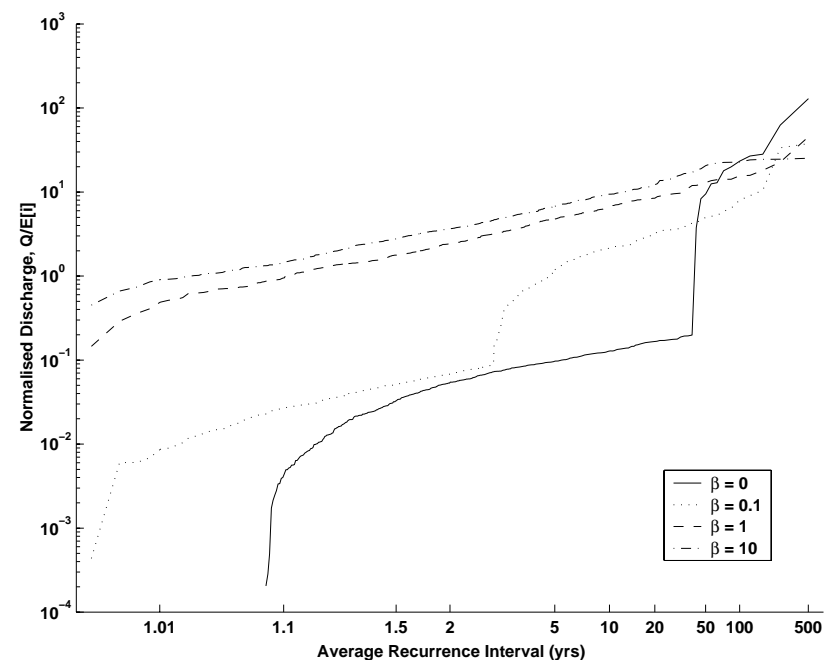

Fig. 7b. The impact of variable soil depth and partial saturation upon flood frequency.

saturation is associated with normalised flood peak values greater than 0 . The impact of variable soil depth is to create shallower-than-average buckets, which will saturate at lower values of $\alpha$ compared to the single-bucket case; the larger the value of $\beta$, the smaller the shallowest bucket depth is, and partial saturation excess onset will occur at progressively lower values of $\alpha$. Figure 7b illustrates the impact of partial saturation upon the flood frequency curve. For small values of $\beta$, which are associated with reductions in the near-stream soil depth, but relatively constant soil depths away from the stream, the incidence of flow cessation is significantly reduced, and (partial) saturation becomes more common. As $\beta$ becomes larger, partial saturation occurs in most or all years, such that flood magnitudes at low return periods are significantly increased. Conversely, extreme flood magnitudes associated with large return periods are reduced by the impact of variable soil depth; in effect, partial saturation increases the efficiency of runoff generation and reduces the overall retention of water in the catchment at any point in time, such that full saturation (i.e. $100 \%$ of the catchment) rarely if ever occurs.

Variable soil depth therefore seems to simplify the behaviour of the flood frequency curve, by reducing or eliminating flow reduction and cessation due to evaporation (region (i)), and allowing a smooth instead of abrupt transition associated with steadily increasing partial saturation instead of binary saturation behaviour. It is crucial to note, however, that this simplifying impact has been exaggerated by the assumed organisation of soil depths within the catchment. By assuming that soil depth monotonically decreases towards the stream, the impact of run-on of surface runoff as it moves down slope is neglected, which would otherwise result in more abrupt transitions in the extent of partial saturation. 


\section{Conclusions}

In the absence of thresholds, runoff is produced by a single mechanism; in this study, subsurface flow. Deviation of flood frequency from rainfall frequency for this single mechanism will be functional upon the characteristic response timescale associated with the runoff generation and routing processes (i.e. attenuation) and non-runoff losses which impact antecedent conditions and runoff recession (e.g., evaporation).

The addition of thresholds introduced a multiplicity of intermittently-active runoff generation pathways; deviation of flood frequency from rainfall frequency will therefore be heterogeneous, with different ranges of return period associated with different regimes of runoff pathway activity. Activation of a given flow pathway is conditional upon threshold exceedence, such that the transition from one regime to another will depend upon the frequency of threshold exceedence. For storage-based thresholds, the relative frequency of threshold exceedance depends upon the value of the threshold itself, properties of rainfall forcing including deterministic and stochastic variability, and non-runoff losses which impact the antecedent condition. The degree of heterogeneity in the flood frequency curve will therefore be maximised where there is a wide discrepancy between the response timescales associated with each runoff mechanism, and where the per-storm frequency of threshold exceedence is greater than 0 but less than 1 .

Temporal variability in storm properties associated with seasonality always has the impact of increasing the magnitude of the flood frequency response associated with a given runoff process, and is likely to increase the frequency of threshold exceedence, relative to a non-seasonal case with the same average storm properties. Spatial variability in landscape properties and climate properties results in spatial variability in the local frequency of threshold exceedence, with the nature of landscape spatial variability being crucial in determining how this impacts upon catchment flood frequency. For decreasing soil depth towards the stream, for example, partial saturation (i.e. threshold exceedance in a temporally-variable proportion of the total catchment area) results in a masking of the impacts of threshold behaviour upon the resulting flood frequency; nonetheless, flood frequency behaviour remains heterogeneous.

Acknowledgements. The authors wish to acknowledge funding support provided through an Australian Research Council (ARC) Discovery Grant awarded to the second author. SESE Reference 053.

Edited by: L. Pfister

\section{References}

Atkinson, S. E., Woods, R. A., and Sivapalan, M.: Climate and landscape controls on water balance model complexity over changing timescales, Water Resour. Res., 38(12), 1314, doi:10.1029/2002WR001487, 2002.

Blöschl, G. and Sivapalan, M.: Process controls on regional flood frequency: coefficient of variation and basin scale, Water Resour. Res., 33(12), 2967-2980, 1997.

Eagleson, P. S.: Dynamics of flood frequency, Water Resour. Res., 8(4), 878-898, 1972.

Gupta, V. K., Castro, S. L., and Over, T. M.: On scaling exponents of spatial peak flows from rainfall and river network geometry, J. Hydrol., 187, 81-104, 1996.

Jothityangkoon, C. and Sivapalan, M.: Temporal scales of rainfallrunoff processes and spatial scaling of flood peaks: Space-time connection through catchment water balance, Adv. Water Resour., 24(9-10), 1015-1036, 2001.

Kiem, A. S., Franks, S. W., and Kuczera, G.: Multi-decadal variability of flood risk, Geophys. Res. Lett., 30(2), 1035, doi:10.1029/2002GL015992, 2003.

Kusumastuti, D. I., Struthers, I., Sivapalan, M., and Reynolds, D. A.: Threshold effects in catchment storm response and the occurrence and magnitude of flood events: implications for flood frequency, Hydrol. Earth Syst. Sci. Discuss., 3, 3239-3277, 2006, http://www.hydrol-earth-syst-sci-discuss.net/3/3239/2006/.

Manabe, S.: Climate and the ocean circulation, 1, The atmospheric circulation and the hydrology of the Earth's surface, Mon. Wea. Rev., 97(11), 739-774, 1969.

Merz, R. and Blöschl, G.: A process typology of regional floods, Water Resour. Res., 39(12), 1340, doi:10.1029/2002WR001952, 2003.

Pattison, A., Ward, J. K. G., McMahon, T. A., and Watson, B. (Eds.): Australian Rainfall and Runoff: Flood Analysis and Design, The Institution of Engineers, Australia, p. 160, 1977.

Robinson, J. S. and Sivapalan, M.: Temporal scales and hydrological regimes: Implications for flood frequency scaling, Water Resour. Res., 33(12), 2981-2999, 1997.

Sivapalan, M., Wood, E. F., and Beven, K. J.: On hydrological similarity 3. A dimensionless flood frequency model using and generalised geomorphologic unit hydrograph and partial area runoff generation, Water Resour. Res., 26(1), 43-58, 1990.

Sivapalan, M. and Blöschl, G.: Transformation of point rainfall to areal rainfall: Intensity-duration-frequency curves, J. Hydrol., 204, 150-167, 1998.

Struthers, I., Hinz, C., and Sivapalan, M.: Conceptual examination of climate-soil controls upon rainfall partitioning in an openfractured soil II. Response to a population of storms, Adv. Water Resour., 30(3), 518-527, doi:10.1016/j.advwatres.2006.04.005, 2007.

Wood, E. F., Lettenmaier, D. P., and Zartarian, V. G.: A land-surface hydrology parameterization with subgrid variability for general circulation models, J. Geophys. Res., 97, 2717-2728, 1992. 